\title{
The discovery of LINERs and a premonition that black holes are found in every galaxy
}

\author{
G. Kauffmann \\ Max Planck Institut für Astrophysik, Karl Schwarzschildstrasse 1, 85741 Garching, Germany \\ e-mail: gamk@mpa-garching.mpg.de
}

The 1980 paper by T. M. Heckman is the standard reference for a type of galactic nucleus called a LINER (low-ionization nuclear emission-line region), which is defined by its spectral line emission. The spectra of these objects typically include line emission from weakly ionized or neutral atoms, such as $\mathrm{O}, \mathrm{O}+, \mathrm{N}+$, and $\mathrm{S}+$. Unlike the more luminous but much rarer Seyfert 2 nuclei, the spectral line emission from strongly ionized atoms, such as $\mathrm{O}++, \mathrm{Ne}++$, and $\mathrm{He}+$, is relatively weak in LINERs. Prior to this paper, a handful of these objects had been studied, and the view was that they constituted a rare class of active galactic nucleus (AGN) in which shock-heating of the gas was likely to play an important role in exciting the line emission. Heckman's paper, which was the third and last in a series published in A\&A and A\&AS in 1980 on the properties of the nuclear regions of nearby galaxies, demonstrated clearly that LINERs are ubiquitous in the nuclei of early type galaxies. The paper was also the first to use the acronym LINER, which now has its own Wikipedia entry!

The survey on which the paper is based consists of a sample of 90 galaxies listed in the Second Reference Catalog of Galaxies (de Vaucouleurs et al. 1976) as brighter than 12th magnitude and north of declination $+40 \mathrm{deg}$. The radio data was obtained using the first set of antennae that were available on the VLA. In the late 1970s, almost all radio interferometry was done in Europe or Australia and most American astronomers had to be content with single-dish observations using the Arecibo or Green Bank telescopes.

Although Heckman's survey only contained a small number of galaxies, the important point is that it was an unbiased study of nuclear activity in a small, but representative sample of nearby galaxies. The sample was selected using only the apparent magnitude of the galaxy as a criterion, hence insuring that it did not favor any particular form of nuclear activity and that it included a wide range of galaxies of different intrinsic luminosities and types. This allowed Heckman to characterize both the LINER phenomenon itself and the properties of LINER host galaxies in considerable detail.

Some of Heckman's main conclusions about the LINER nuclear activity in galaxies are: 1) that the total luminosities of the line-emitting regions are low $\left(10^{38}-10^{40} \mathrm{erg} \mathrm{s}^{-1}\right)$, so more similar to giant HII regions than the nuclei of Seyferts or quasars; 2) that the widths of the emission lines are similar to the lines originating in the narrow-line gas in Seyferts; 3) that there is no evidence of a featureless blue continuum that indicates the presence of an accretion disk in these objects; and 4) that LINERs are frequently associated with compact nuclear radio sources.

His study of the correlation between LINER activity and host galaxy properties showed that: 1) LINERs occur in a third of all galaxies in the local Universe; 2) that the LINER fraction peaks in galaxies with early-type (E,S0,Sa) morphologies; 3$)$ that there is no correlation between LINER activity and the HI gas content of the galaxies; and 4) that LINERs with compact nuclear radio sources occurred more frequently in the most luminous galaxies in the sample.

The second part of Heckman's paper is devoted to the still thorny question of the physical origin of the line emission in these objects. Heckman's paper presents a detailed overview of all the possibilities, but shock-wave models are clearly the favored interpretation. The common view today is that most LINERs are photo-ionized rather than shock-heated (see Ho 2008 for an extensive recent discussion of this subject). The early evidence of shocks was largely based on the high electron temperatures that had been derived from the ratio of the [OIII] $\lambda 4363$ line and the [OIII] $\lambda 5007$ lines in a few of the brightest objects. These ratios were not consistent with photoionization models. In addition, the lack of any detectable nonstellar ultraviolet continuum and the relatively large widths of the emission lines all seemed to favor the shock hypothesis. It was later demonstrated that the high electron temperatures were incorrect because of erroneous measurements. The currently popular explanation for the lack of non-thermal UV continuum is that LINERs are experiencing a different mode of accretion to the higher luminosity Seyferts or quasars. Once the accretion rate onto the black hole drops below a certain critical value, the broad line region and the obscuring torus disappear, and the accretion disk transforms into a structure consisting of an inner, radiatively inefficient accretion flow and a truncated outer thin disk (see Ho 2008 and references therein).

Nevertheless it remains an open question as to what extent LINERs constitute a homogeneous population of objects with only one single line-excitation mechanism. Heckman's current view is that the situation is confused because there may be several different phenomena that are all called LINER. At the very lowest luminosities, it has been claimed that photoionization by post-AGB stars will lead to LINER-like emission in all early-type galaxies. This would set the floor at the very lowest 
luminosities. LINER-like emission also occurs in cooling flow nebulae. This is clearly not produced by AGN photoionization, but the actual mechanism is unclear. Finally, there are starburst galaxies with LINER-like ("transition") spectra where some combination of photo-ionization by hot stars and shock heating by $\mathrm{SNe}$ and galactic winds is likely. Nevertheless, Heckman believes that LINERs produced by photoionization from the radiation from a black hole are likely to dominate by number in unbiased surveys of nearby galaxies. Rather little is known about LINERs in the more distant Universe, but this is because many of the key emission lines are redshifted out of the optical into the near-infrared, where they become much more difficult to observe. High-redshift galaxies are much more active, and most are forming stars at rates characteristic of nearby starburst systems. It may well turn out that the shock models again become fashionable once near-infrared spectra in large samples of high-redshift galaxies finally become available.

At low redshifts, large spectroscopic surveys, in particular the Sloan Digital Sky Survey, have led to a thousandfold increase in the samples of low-luminosity AGN available for study. This has led to the discovery that LINERs and Seyferts do indeed segregate cleanly in emission-line diagnostic diagrams involving lower-ionization lines, such as [OI] (Kewley et al. 2006). Before, it had been hypothesized that LINERs and Seyferts together form a continuous sequence of increasing ionization parameter. Another important recent finding from large surveys is that the LINER and the nuclear radio-source phenomena are independent of each other (Best et al. 2005). Since then, Heckman and I wrote a paper showing that SDSS data support a picture in which early-type galaxies with LINERs are fueled by mass loss from evolved stars (Kauffmann \& Heckman 2009), another idea that has been around since the 1980s (Norman \& Scoville 1988).

Perhaps the most enduring legacy of Heckman's paper, however, is that it actually foreshadowed the much later discovery that black holes are a generic feature of all galactic bulges. The idea that black holes and galaxies form and evolve together is now taken for granted. Theoreticians such as myself routinely construct so-called "unified models" in which black holes and bulges form together as structure in the Universe grows through gravitational instability and hierarchical clustering. In the most recent incarnations of such models, the black hole is actually responsible for controlling the growth of the galaxy and eventually blowing out its gas and shutting down star formation (Di Matteo et al. 2005). In the 1980s, the idea that black holes and galaxies might be so intimately linked was a concept that had not yet taken root in the minds of most astronomers working on galaxies or on AGN. Viewed from this perspective, Heckman's paper is quite remarkable for its prescience.

\section{References}

Best, P. N., Kauffmann, G., Heckman, T. M., \& Ivezić, Ž. 2005, MNRAS, 362, 9

De Vaucouleurs, G., De Vaucouleurs, A., \& Corwin, H. G. 1976, The Second Reference Catalog of Bright Galaxies (Austin: University of Texas)

Di Matteo, T., Springel, V., \& Hernquist, L. 2005, Nature, 433, 604

Heckman, T. M. 1980, A\&A, 87, 152

Ho, L. C. 2008, ARA\&A, 46, 475

Kauffmann, G., \& Heckman, T. M. 2009, MNRAS, in press [arXiv:0812.1224]

Kewley, L. J., Groves, B., Kauffmann, G., \& Heckman, T. 2006, MNRAS, 372, 961

Norman, C., \& Scoville, N. 1988, ApJ, 332, 124 